\title{
A Fistula Where? Left Anterior Descending to Pulmonary Artery Fistula
}

\author{
Samantha Gelman ${ }^{\mathrm{a}, \mathrm{b}}$, Andrew Benin ${ }^{\mathrm{a}}$, Javad Savoj ${ }^{\mathrm{a}}$, Rajesh Gulati ${ }^{\mathrm{a}}$, \\ Kaustubh Patankara ${ }^{\mathrm{a}}$ Patrick $\mathrm{Hu}^{\mathrm{a}}$
}

\begin{abstract}
Fistulas are abnormal passageways between two organs or vessels that usually do not connect. Coronary artery fistulas occur when one of the coronary arteries is connected to either a heart chamber or another blood vessel. This case presents a coronary artery fistula which may or may not be impacting the patient's heart function. A 69-year-old male with multiple comorbidities presents with a chief complaint of shortness of breath. Catheterization reveals an anomalous left anterior descending to pulmonary artery fistula without a step up in oxygen saturation at the level of the pulmonary artery. Surgical management was deferred as this fistula was not deemed to contribute to the patient's declining cardiac function. Operative management versus embolization would be a feasible alternative for patients who are symptomatic secondary to the coronary artery fistula and remains to be controversial in patients who are asymptomatic. Given the possibility of these fistulae eventually causing symptoms it would be practical to close them early on before symptoms arise or before size of the fistulae becomes an issue. Further research should be conducted to understand the management strategies for patients who present with coronary artery fistulas.
\end{abstract}

Keywords: Coronary vessel anomaly; Coronary angiography; Coronary artery fistula

\section{Introduction}

Fistulas in general are abnormal passageways between two organs or vessels that usually do not connect. They can develop anywhere but they are rarely seen in the heart. Coronary artery fistulas occur when one of the coronary arteries is connected to

Manuscript submitted June 11, 2020, accepted June 30, 2020

Published online August 28, 2020

${ }^{a}$ Graduate Medical Education Program, Department of Medicine, Riverside Community Hospital/Hospital Corporation of America (HCA), Riverside, CA 92501, USA

${ }^{\mathrm{b} C}$ Corresponding Author: Samantha Gelman, Graduate Medical Education Program, Department of Medicine, Riverside Community Hospital/ Hospital Corporation of America (HCA), 4445 Magnolia Avenue, Riverside, CA 92501, USA. Email: lakhtarniksamantha@yahoo.com

doi: https://doi.org/10.14740/jmc3527 either a heart chamber or another blood vessel. They generally occur when one of the coronary arteries fail to form properly likely due to an underlying congenital defect. However, this can also occur after birth, following heart surgery or post infection that causes weakening of the artery or the heart, and even after a traumatic injury to the cardiac tissue. This case presents a coronary artery fistula which may or may not be impacting the patient's heart function [1].

\section{Case Report}

A 69-year-old male with a past medical history of chronic obstructive pulmonary disease (COPD), atrial fibrillation on anticoagulation, and heart failure with reduced ejection fraction in the setting of longstanding atrial fibrillation presented with a chief complaint of shortness of breath. The patient was subsequently admitted for heart failure exacerbation. Physical exam findings were unremarkable. He was found to have an ejection fraction of $20 \%$ on echocardiogram which has worsened from his prior ejection fraction of $35 \%$. He was treated with an ace inhibitor, beta blockers, spironolactone and another diuretic. Catheterization findings demonstrated a left ventricular ejection fraction of $20 \%$ without evidence of coronary artery disease with findings of an anomalous left anterior descending (LAD) to pulmonary artery fistula without any step up in oxygen saturation at the level of the pulmonary artery (Figs. 1 and 2). The patient also was found to have a $100 \%$ occlusion of the bilateral iliac arteries.

This patient was previously found to have this coronary artery fistula and was medically managed. Cardiothoracic surgery was consulted on this admission and recommended that no surgical intervention be pursued at this time with continued medical management or consideration of coiling of the fistula if it is the likely cause for the decline in his heart function. The patient's shortness of breath improved with diuresis. The patient was discharged with goal directed medical therapy for heart failure with reduced ejection fraction for at least 3 months and outpatient follow-up for automatic implantable cardioverter defibrillators (AICD) evaluation.

\section{Discussion}

Current research shows that this is a rare congenital anomaly 


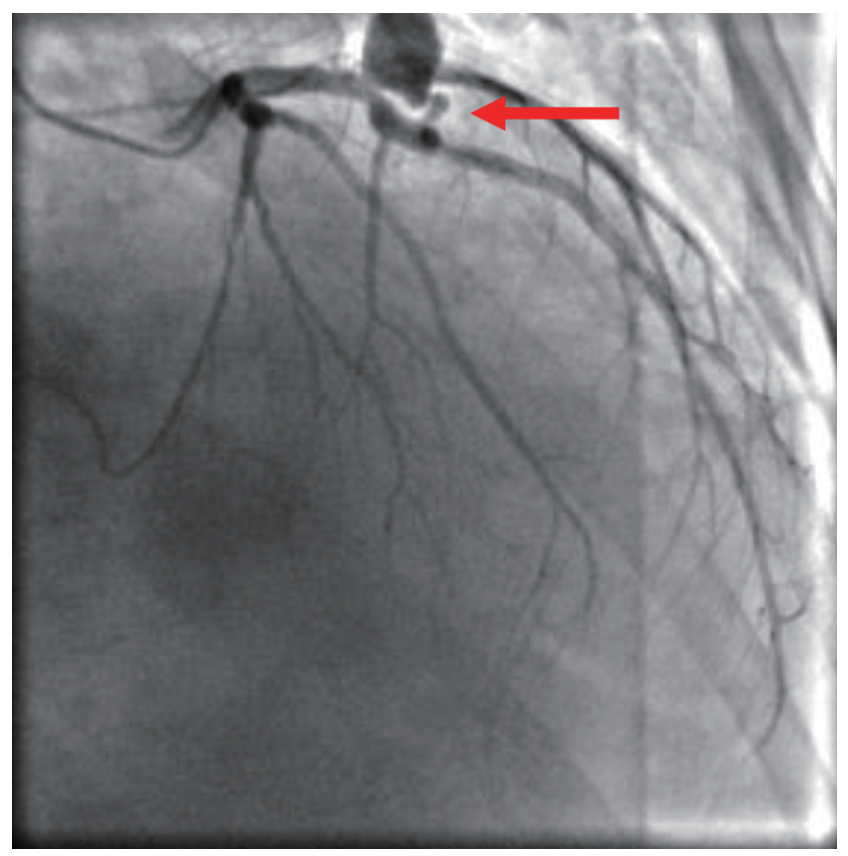

Figure 1. Coronary artery fistula. Angiography shows the LAD to pulmonary artery fistula (arrow). LAD: left anterior descending.

that occurs in $0.2-0.4 \%$ of congenital cardiac anomalies. With the advent of computed tomography (CT) angiography $0.9 \%$ of individuals have been incidentally diagnosed with a coronary artery fistula. It can cause myocardial ischemia, sudden cardiac death, ischemic cardiomyopathy, papillary muscle rupture, secondary valvular disease, premature atherosclerosis, among others [2]. Most patients are asymptomatic and if they are associated with other conditions such as, coronary artery disease or heart failure they may present with symptoms of angina as well as, dyspnea. There is no specific treatment modality for coronary fistulas but there have been reports of surgical intervention as well as, trans-catheter embolization [3].

Operative management versus embolization would be a feasible alternative for patients who are symptomatic secondary to the coronary artery fistula and remains to be controversial in patients who are asymptomatic. A study conducted in 2017, concluded that out of 21 adult patients that underwent surgical closure with ligation of the coronary artery fistula none were found to have operative or hospital mortality. The hospital course was complicated for three of these patients due to the development of postoperative atrial fibrillation and one patient who developed cardiac tamponade [4].

In this case, there was a lack of shunting demonstrated during the angiography pointing against the LAD to pulmonary artery fistula as the culprit for the patient's symptoms and recent worsening of cardiac function. The etiology for his heart failure is likely attributed to tachycardia induced cardiomyopathy in the setting of atrial fibrillation and he would benefit from aggressive goal directed medical therapy. This case illustrates the importance of diagnosing rare conditions with the increasing use of angiography and how this may implement

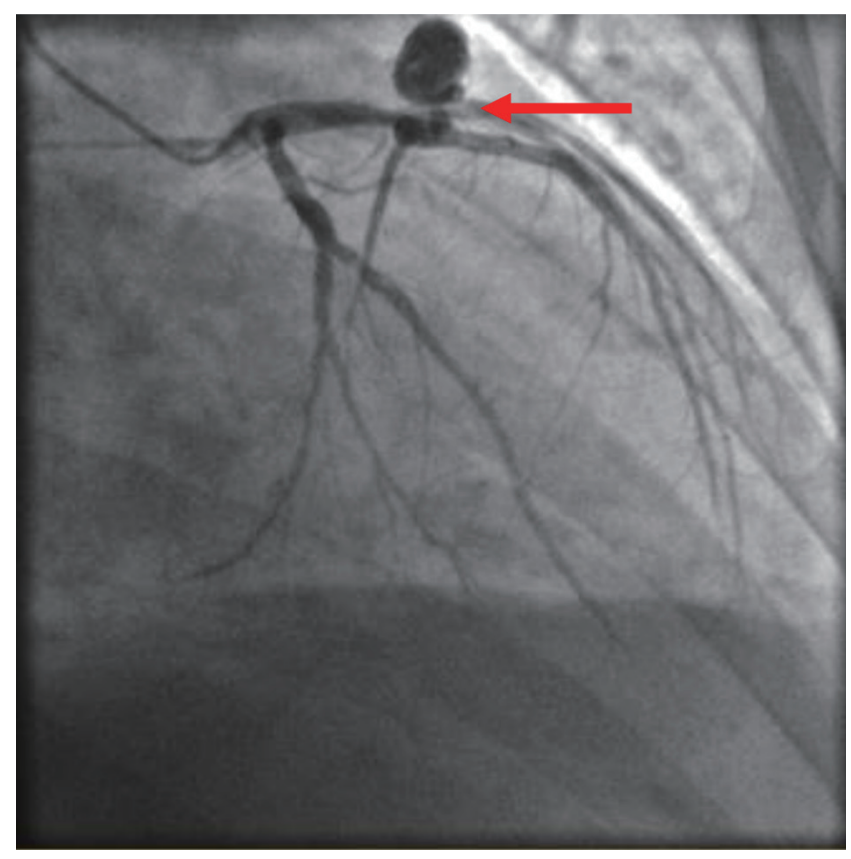

Figure 2. Coronary artery fistula. A second view of angiography of LAD to pulmonary artery fistula (arrow). LAD: left anterior descending.

the health of an individual.

\section{Conclusions}

The consensus seems to point to operative management in symptomatic patients if the fistula was the underlying etiology for worsening heart function or any other related sequelae. However, there has been controversy regarding size and location of the fistulae in asymptomatic individuals with respect to surgical management [5]. Given the possibility of these fistulae eventually causing symptoms it would be practical to close them early on before symptoms arise or before size of the fistulae becomes an issue. Further research should be conducted to understand the management strategies for patients who present with coronary artery fistulas and determine in whom further intervention may be necessary depending on whether other underlying comorbid conditions are present or absent. It would also be beneficial to monitor the long-term outcomes in cardiac function when comparing those who received operative management versus those who didn't.

\section{Acknowledgments}

None to declare.

\section{Financial Disclosure}

The authors have no relevant financial disclosure pertaining to this manuscript. 


\section{Conflict of Interest}

The authors have reported that they have no conflict of interest relevant to the content of this paper to disclose.

\section{Informed Consent}

Informed consent was not needed for this case.

\section{Author Contributions}

Gelman, S; Benin, A; Savoj, J presented the idea, collected data, conducted additional research, obtaining angiography film, editing of manuscript. Gelman, S wrote the manuscript. Patankar, K supervised the project. Gulati, R provided critical feedback. Hu, Patrick provided supervision, editing of manuscript. All authors discussed the results and contributed to the final manuscript.

\section{Data Availability}

The authors declare that data supporting the findings of this study are available within the article.

\section{References}

1. Ibrahim MF, Sayed S, Elasfar A, Sallam A, Fadl M, Al Baradai A. Coronary fistula between the left anterior descending coronary artery and the pulmonary artery: Two case reports. J Saudi Heart Assoc. 2012;24(4):253256.

2. Sherif K, Mazek H, Otahbachi M. Coronary Artery and Pulmonary Artery Fistula. JACC: Case Reports. 2020;2(2):286-288.

3. Chowdhury MA, Chikodi S, Moza A, Bieszczad JE, Eltahawy E. Coronary Artery to Pulmonary Artery Fistula With Concomitant Atrial Fibrillation. Cath Lab Digest. 2018;26(9). www.cathlabdigest.com/article/CoronaryArtery-Pulmonary-Artery-Fistula-Concomitant-AtrialFibrillation.

4. Albeyoglu S, Aldag M, Ciloglu U, Sargin M, Oz TK, Kutlu H, Dagsali S. Coronary arteriovenous fistulas in adult patients: surgical management and outcomes. Braz J Cardiovasc Surg. 2017;32(1):15-21.

5. Challoumas D, Pericleous A, Dimitrakaki IA, Danelatos C, Dimitrakakis G. Coronary arteriovenous fistulae: a review. Int J Angiol. 2014;23(1):1-10. 